\title{
KRULL DIMENSION AND GENERALIZED FRACTIONS
}

\author{
by M. A. HAMIEH and R. Y. SHARP
}

(Received 30th April 1984)

\section{Introduction}

Let $R$ be a (commutative Noetherian) local ring (with identity) having maximal ideal $\mathrm{m}$ and dimension $d \geqq 1$. It is shown in $[5,3.6]$ that the local cohomology module $H_{\mathrm{m}}^{d}(R)$ may be described as a module of generalized fractions: if $x_{1}, \ldots, x_{d}$ is a system of parameters for $R$, then $H_{\mathrm{m}}^{d}(R) \cong U(x)_{d+1}^{-d-1} R$, where $U(x)_{d+1}$ is the triangular subset $[4,2.1]$ of $R^{d+1}$ given by

$$
\begin{array}{r}
U(x)_{d+1}=\left\{\left(x_{1}^{\alpha_{1}}, \ldots, x_{d}^{\alpha_{d}}, 1\right): \text { there exists } j \text { with } 0 \leqq j \leqq d\right. \text { such that } \\
\left.\qquad \alpha_{1}, \ldots, \alpha_{j} \in \mathbb{N} \text { and } \alpha_{j+1}=\cdots=\alpha_{d}=0\right\} .
\end{array}
$$

Now it is well known (see [1, Proposition 6.4(4)] or $[2,2.2]$ ) that $H_{\mathrm{m}}^{d}(R) \neq 0$. Thus it is possible to find a triangular subset $U$ of $R^{d+1}$ for which $U^{-d-1} R \neq 0$. It seems natural to ask whether there is any upper bound for the set of positive integers $n$ for which there exists a triangular subset $V$ of $R^{n}$ with $V^{-n} R \neq 0$. The purpose of this note is to prove a theorem that relates Krull dimension and modules of generalized fractions, and it will follow from the theorem that $d+1$ is itself a bound of the specified type.

Our main result will not need any Noetherian hypothesis and so we shall work throughout over a commutative ring $A$ (with identity); $M$ will denote an $A$-module. When $M \neq 0, \operatorname{dim} M$ (or $\operatorname{dim}_{A} M$ ) will denote the dimension of $M$, that is the supremum of lengths of chains of prime ideals of $\operatorname{Supp}(M)$ if this exists, and $\infty$ otherwise. For $\mathfrak{p} \in \operatorname{Supp}(M)$, the $M$-height of $\mathfrak{p}$, denoted $\mathrm{ht}_{M} \mathfrak{p}$, is defined to be $\operatorname{dim}_{A_{\mathrm{p}}} M_{\mathrm{p}}$.

We shall use the terminology and notation of $[4,5]$ concerning modules of generalized fractions and related concepts.

\section{Some technical results about generalized fractions}

We shall need the fact that ordinary fraction formation "commutes", in a certain sense, with formation of generalized fractions. This fact is mentioned in [3], where it is obtained as a consequence of O'Carroll's elegant description of certain modules of generalized fractions in terms of ordinary modules of fractions. However, O'Carroll mentions that this fact was proved earlier by the second of the two present authors by direct computation: the calculations are included here, as they are not completely obvious and provide a short and unsophisticated proof of a basic fact. 
Proposition 2.1. Let $U$ be a triangular subset of $A^{n}$ (where $n \in \mathbb{N}$ ) and let $S$ be a multiplicatively closed subset of $A$. Let $\phi: A \rightarrow S^{-1} A$ denote the natural ring homomorphism, and set

$$
U_{S}=\left\{\left(\phi\left(a_{1}\right), \ldots, \phi\left(a_{n}\right)\right):\left(a_{1}, \ldots, a_{n}\right) \in U\right\}
$$

Then $U_{S}$ is a triangular subset of $\left(S^{-1} A\right)^{n}$ and there is an isomorphism of $S^{-1} A$-modules $\psi: S^{-1}\left(U^{-n} M\right) \rightarrow\left(U_{S}\right)^{-n}\left(S^{-1} M\right)$ which is such that, for $m \in M,\left(u_{1}, \ldots, u_{n}\right) \in U$ and $s \in S$,

$$
\psi\left(\frac{m}{\left(u_{1}, \ldots, u_{n}\right)} / s\right)=\frac{m / s}{\left(\phi\left(u_{1}\right), \ldots, \phi\left(u_{n}\right)\right)} .
$$

Proof. It is routine to check that $U_{S}$ is a triangular subset of $\left(S^{-1} A\right)^{n}$ and that there is an $S^{-1} A$-epimorphism $\psi: S^{-1}\left(U^{-n} M\right) \rightarrow\left(U_{S}\right)^{-n}\left(S^{-1} M\right)$ given by the formula in the statement of the proposition: the only non-obvious matter is the proof that $\psi$ is injective.

For $\mathbf{H}=\left[h_{i j}\right] \in D_{n}(A)$, set $\phi(\mathbf{H})=\left[\phi\left(h_{i j}\right)\right], \in D_{n}\left(S^{-1} A\right)$. Note that $|\phi(\mathbf{H})|=\phi(|\mathbf{H}|)$. Let $m \in M,\left(u_{1}, \ldots, u_{n}\right) \in U$ and $s \in S$ be such that

$$
(m / s) /\left(\phi\left(u_{1}\right), \ldots, \phi\left(u_{n}\right)\right)=0
$$

in $\left(U_{S}\right)^{-n}\left(S^{-1} M\right)$. Thus there exist $\left(v_{1}, \ldots, v_{n}\right) \in U$ and $\mathbf{L} \in D_{n}\left(S^{-1} A\right)$ such that

$$
\mathbf{L}\left[\phi\left(u_{1}\right) \ldots \phi\left(u_{n}\right)\right]^{T}=\left[\phi\left(v_{1}\right) \ldots \phi\left(v_{n}\right)\right]^{T}
$$

and

$$
|\mathbf{L}|(m / s) \in\left(\sum_{i=1}^{n-1} S^{-1} A\left(\phi\left(v_{i}\right)\right)\right) S^{-1} M
$$

Now there exist $\left(w_{1}, \ldots, w_{n}\right) \in U$ and $\mathbf{H}, \mathbf{K} \in D_{n}(A)$ such that

$$
\mathbf{H}\left[\begin{array}{lll}
u_{1} & \ldots & u_{n}
\end{array}\right]^{T}=\left[\begin{array}{llll}
w_{1} & \ldots & w_{n}
\end{array}\right]^{T}=\mathbf{K}\left[\begin{array}{lll}
v_{1} & \ldots & v_{n}
\end{array}\right]^{T} .
$$

Hence $\phi(\mathbf{H})\left[\begin{array}{llll}\phi\left(u_{1}\right) & \ldots & \phi\left(u_{n}\right)\end{array}\right]^{T}=\left[\begin{array}{llll}\phi\left(w_{1}\right) & \ldots & \phi\left(w_{n}\right)\end{array}\right]^{T}=\phi(\mathbf{K})\left[\begin{array}{llll}\phi\left(v_{1}\right) & \ldots & \phi\left(v_{n}\right)\end{array}\right]^{T}$. Let $\mathbf{D}=$ $\operatorname{diag}\left(w_{1}, \ldots, w_{n}\right)$, so that $\phi(\mathbf{D})=\operatorname{diag}\left(\phi\left(w_{1}\right), \ldots, \phi\left(w_{n}\right)\right)$. It is now easy to use $[4,2.2$ and 2.3$]$ to see that

$$
|\phi(\mathbf{D}) \phi(\mathbf{H})|(m / s) \in\left(\sum_{i=1}^{n-1} S^{-1} A\left(\phi\left(w_{i}\right)\right)^{2}\right) S^{-1} M
$$

It follows that, for some $t \in S$,

$$
t|\mathbf{D H}| m \in\left(A w_{1}^{2}+\cdots+A w_{n-1}^{2}\right) M .
$$


Since $\mathbf{D H}\left[u_{1} \ldots u_{n}\right]^{T}=\left[w_{1}^{2} \ldots w_{n}^{2}\right]^{T}\left(\right.$ and $\left.\left(w_{1}^{2}, \ldots, w_{n}^{2}\right) \in U\right)$, we see from $[4,(3.3)(i i)]$ that

$$
\frac{t m}{\left(u_{1}, \ldots, u_{n}\right)}=0
$$

in $U^{-n} M$. It follows that

$$
\frac{m}{\left(u_{1}, \ldots, u_{n}\right)} / s=0
$$

in $S^{-1}\left(U^{-n} M\right)$. Hence $\psi$ is an isomorphism.

We shall also need the following lemma about modules of generalized fractions.

Lemma 2.2. Let $m, n \in \mathbb{N}$ with $m<n$. Let $U$ be a triangular subset of $A^{n}$ and let $V$ be the restriction $[4,3.6]$ of $U^{\prime}$ to $A^{m}$. Let $x \in \dot{M},\left(u_{1}, \ldots, u_{n}\right) \in U$ be such that $x /\left(u_{1}, \ldots, u_{n}\right) \neq 0$ in $U^{-n} M$. Then $x /\left(u_{1}, \ldots, u_{m}\right) \neq 0$ in $V^{-m} M$.

Proof. Let $\bar{U}$ (respectively $\bar{V}$ ) be the expansion $[4,3.2]$ of $U$ (respectively $V$ ). Since $\bar{V}$ is the restriction of $\bar{U}$ to $A^{m}$, it follows from $[4,3.2]$ that we may assume that $U$ (and hence $V$ ) is expanded. Also, we may assume that $m=n-1$.

Suppose that $x /\left(u_{1}, \ldots, u_{n-1}\right)=0$. Then there exist $\left(v_{1}, \ldots, v_{n-1}\right) \in V$ and $\mathbf{H} \in D_{n-1}(A)$ such that $\mathbf{H}\left[\begin{array}{lll}u_{1} & \ldots & u_{n-1}\end{array}\right]^{T}=\left[\begin{array}{llll}v_{1} & \ldots & v_{n-1}\end{array}\right]^{T}$ and $|\mathbf{H}| x \in\left(A v_{1}+\cdots+A v_{n-2}\right) M$. Now $\mathbf{K}\left[\begin{array}{llll}u_{1} & \ldots & u_{n-1} & 1\end{array}\right]^{T}=\left[\begin{array}{llll}v_{1} & \ldots & v_{n-1} & 1\end{array}\right]^{T}$, where

$$
\mathbf{K}=\left[\begin{array}{l|l}
\mathbf{H} & 0 \\
\hline 0 & 1
\end{array}\right], \in D_{n}(A),
$$

and $|\mathbf{K}| x=|\mathbf{H}| x \in\left(A v_{1}+\cdots+A v_{n-1}\right) M$. Since $U$ is expanded, we note that $\left(u_{1}, \ldots, u_{n-1}, 1\right)$, $\left(v_{1}, \ldots, v_{n-1}, 1\right) \in U$. Hence

$$
\frac{x}{\left(u_{1}, \ldots, u_{n-1}, 1\right)}=0
$$

in $U^{-n} M$. Hence $u_{n} x /\left(u_{1}, \ldots, u_{n-1}, u_{n}\right)=0$, and so, by $[5,2.1], x /\left(u_{1}, \ldots, u_{n-1}, u_{n}\right)=0$. This contradiction completes the proof.

\section{The results}

The dimension of a non-zero $A$-module $M$ and the $M$-height of a prime ideal $\mathfrak{p} \in \operatorname{Supp}(M)$ were defined in the Introduction. For a triangular subset $U$ of $A^{n}$ (where $n \in \mathbb{N}$ ) and a $q \in \operatorname{Spec}(A)$, we shall write $U_{q}$ instead of the $U_{A \mid q}$ of 2.1 .

Theorem 3.1. Let $U$ be a triangular subset of $A^{n}$ (where $\left.n \in \mathbb{N}\right)$. Then

$$
\operatorname{Supp}\left(U^{-n} M\right) \subseteq\left\{\mathfrak{p} \in \operatorname{Supp}(M): \mathrm{ht}_{M} \mathfrak{p} \geqq n-1\right\} .
$$


Proof. It is easy to see that $\operatorname{Supp}\left(U^{-n} M\right) \subseteq \operatorname{Supp}(M)$. We prove the claim by induction on $n$, there (now) being nothing to do when $n=1$. Thus we suppose that $n>1$ and that the result has been proved for triangular subsets of $A^{n-1}$.

Let $p \in \operatorname{Supp}\left(U^{-n} M\right)$, so that there exist $m \in M$ and $\left(u_{1}, \ldots, u_{n}\right) \in U$. such that $p \supseteq \operatorname{ann}(x)$, where $x=m /\left(u_{1}, \ldots, u_{n}\right)$. Let $V$ be the restriction of $U$ to $A^{n-1}$, and let $y=m /\left(u_{1}, \ldots, u_{n-1}\right)$ in $V^{-n+1} M$. By 2.2 , ann $(y) \subseteq$ ann $(x)$, and so, by the inductive hypothesis, $\mathfrak{p} \in \operatorname{Supp}(M)$ and $\mathrm{ht}_{M} \mathrm{p} \geqq n-2$. Suppose that $\mathrm{ht}_{M} \mathrm{p}=n-2$.

In view of the inductive hypothesis, this implies that $\operatorname{Supp}_{A_{\mathrm{p}}}\left(\left(V^{-n+1} M\right)_{\mathrm{p}}\right)=\left\{\mathfrak{p} A_{\mathrm{p}}\right\}$. Hence $\sqrt{ }\left(\operatorname{ann}_{A_{p}}(y / 1)\right)=\mathfrak{p} A_{p}$. Now $u_{n-1} \in \operatorname{ann}(x) \subseteq \mathfrak{p}$ and so, by 2.1 , there exists $t \in \mathbb{N}$ such that

$$
\phi\left(u_{n-1}\right)^{t}\left[(m / 1) /\left(\phi\left(u_{1}\right), \ldots, \phi\left(u_{n-1}\right)\right)\right]=0
$$

where $\phi: A \rightarrow A_{\mathfrak{p}}$ denotes the natural ring homomorphism. We now use [5,2.1] and Proposition 2.1 again to see that $y / 1=0$, a contradiction. Hence $\mathrm{ht}_{M} \mathfrak{p} \geqq n-1$.

This completes the inductive step and the theorem follows by induction.

Corollary 3.2. $U^{-n} M=0$ whenever $U$ is a triangular subset of $A^{n}$ and the integer $n$ satisfies $n>\operatorname{dim} M+1$. (We adopt the convention that the zero $A$-module has dimension -1.)

If we combine the above with the remarks in the Introduction and use 2.1 again, we can obtain the following.

Corollary 3.3. Suppose that $A$ is (non-trivial and) Noetherian of finite dimension $d$. Then $d+1$ is the greatest integer $i$ for which there exists a triangular subset $U$ of $A^{i}$ such that $U^{-i} A \neq 0$.

Results concerning heights of prime ideals tend to be of more interest when the ring is Noetherian, and we shall assume that $A$ is Noetherian henceforth. Let $U$ be a triangular subset of $A^{n}$, and let $\left(u_{1}, \ldots, u_{n}\right) \in U$. Consider the generalized fraction $z=1 /\left(u_{1}, \ldots, u_{n}\right)$ of $U^{-n} A$. The question of under what conditions $z$ is non-zero is interesting. It follows from a corollary $[6,3.15]$ of the exactness theorem that $z \neq 0$ if $U$ consists of poor $A$ sequences and $\sum_{i=1}^{n-1} A u_{i}$ is a proper ideal; in these circumstances, $\sum_{i=1}^{n-1} A u_{i}$ has height $n-1$. More generally, for certain choices of ( $A$ and) $U$, the question is related to the Monomial Conjecture: see [5,4.1]. Note also that if $S$ is a multiplicatively closed subset of $A$ and, for $s \in S, 1 / s \neq 0$ in $S^{-1} A$, then $s$ is not nilpotent, and so there is a prime ideal $\mathfrak{p}$ of $A$ (necessarily containing 0 and having ht $\mathfrak{p} \geqq 0$ ) such that $s \notin \mathfrak{p}$. We may use 3.1 to obtain a generalization of the latter statement.

Corollary 3.4. Suppose that $A$ is Noetherian, that $n \in \mathbb{N}$, that $U$ is a triangular subset of $A^{n}$ and that $\left(u_{1}, \ldots, u_{n}\right) \in U$ is such that $1 /\left(u_{1}, \ldots, u_{n}\right) \neq 0$ in $U^{-n} A$. Then, for each $i=0, \ldots, n-1$ there is a prime ideal $\mathfrak{p}$ having $\mathrm{ht} \mathfrak{p} \geqq i$ such that

$$
\sum_{j=1}^{i} A u_{j} \subseteq \mathfrak{p} \quad \text { but } \quad u_{i+1} \notin \mathfrak{p}
$$




\section{Notes.}

(i) $\sum_{j=1}^{0} A u_{j}$ is to be interpreted as 0 .

(ii) When $A$ is a catenary domain the conclusion implies that, for each $i=0, \ldots, n-1$, there is a prime ideal $\mathfrak{p}^{\prime}$ of height $i$ such that $\sum_{j=1}^{i} A u_{j} \subseteq \mathfrak{p}^{\prime}$ but $u_{i+1} \notin \mathfrak{p}^{\prime}$.

(iii) The referee has pointed out that the conclusion of 3.4 is still valid even if the hypothesis that $A$ be Noetherian is deleted.

Proof. In view of 2.2 , we may assume that $i=n-1$. Let $x=1 /\left(u_{1}, \ldots, u_{n-1}, u_{n}\right), \neq 0$. Then, by 3.1 and $[4,3.3($ ii) $)$,

$$
\operatorname{Supp}(A x) \subseteq\left\{\mathfrak{p} \in \operatorname{Spec}(A): \sum_{j=1}^{n-1} A u_{j} \subseteq \mathfrak{p} \text { and ht } \mathfrak{p} \geqq n-1\right\} \text {. }
$$

Suppose that $u_{n} \in \mathfrak{p}$ for every prime ideal $\mathfrak{p}$ of $A$ of height at least $n-1$ that contains $\sum_{j=1}^{n-1} A u_{j}$. Then $u_{n} \in \sqrt{ }(\operatorname{ann}(x))$, and so there exists $t \in \mathbb{N}$ such that $u_{n}^{t} x=0$. Hence

$$
\frac{u_{n}^{t}}{\left(u_{1}, \ldots, u_{n-1}, u_{n}\right)}=0 \text {. }
$$

It follows from $[5,2.1]$ that $x=0$. With this contradiction, the proof is complete.

\section{REFERENCES}

1. A. Grothendieck, Local cohomology (Lecture Notes in Mathematics 41, Springer, Berlin, 1967).

2. I. G. Macdonald and R. Y. Sharp, An elementary proof of the non-vanishing of certain local cohomology modules, Quart. J. Math. Oxford (2), 23 (1972), 197-204.

3. L. O'CARroll, On the generalized fractions of Sharp and Zakeri, J. London Math. Soc. (2), 28 (1983), 417-427.

4. R. Y. Sharp and H. Zakerl, Modules of generalized fractions, Mathematika, 29 (1982), $32-41$.

5. R. Y. SHARP and H. ZAKERI, Local cohomology and modules of generalized fractions, Mathematika, 29 (1982), 296-306.

6. R. Y. Sharp and H. Zakeri, Modules of generalized fractions and balanced big CohenMacaulay modules, Commutative algebra: Durham 1981 (London Mathematical Society Lecture Notes 72, ed. R. Y. Sharp, Cambridge University Press, Cambridge, 1982), pp. 61-82.

Department of Pure Mathematics

UNIVERSITY OF SHEFFIELD

SHEFFIELD S3 7RH 